Author affiliations and support information (if applicable) appear at the end of this article.

Published at jco.org on October 2, 2017.

The opinions expressed in this article are those of the authors and do not necessarily reflect the views or policies of the authors' affiliated institutions.

Corresponding author: Lia Gore, MD, University of Colorado Comprehensive Cancer Center, Center for Cancer and Blood Disorders, Children's Hospital Colorado, 13123 East 16th Ave, Box B115, Aurora, CO 80045; e-mail: lia.gore@ ucdenver.edu.

(c) 2017 by American Society of Clinical Oncology

0732-183X/17/3533w-3781w/\$20.00

\title{
Modernizing Clinical Trial Eligibility: Recommendations of the American Society of Clinical Oncology-Friends of Cancer Research Minimum Age Working Group
}

Lia Gore, S. Percy Ivy, Frank M. Balis, Eric Rubin, Katherine Thornton, Martha Donoghue, Samantha Roberts, Suanna Bruinooge, Jennifer Ersek, Nancy Goodman, Caroline Schenkel, and Gregory Reaman

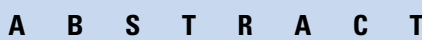

\section{Purpose}

Children have historically been excluded from first-in-human studies of promising new cancer drugs and later phase adult clinical trials. Delays in evaluation may result in off-label use without dosing information as the only access to new drugs. A multistakeholder workshop was convened in May 2016 by ASCO and Friends of Cancer Research to identify opportunities for when it would be scientifically appropriate to expand trial eligibility to include children younger than age 18 years in first-in-human and other adult cancer clinical trials.

\section{Methods}

This group convened experts from academia, government, and industry to review barriers to enrolling children and adolescents in oncology clinical trials. We evaluated the historical context, published literature, regulatory considerations, and myriad risks and benefits associated with lowering the age of enrollment on oncology clinical trials.

\section{Results}

We conclude that many of the historical concerns about including children early in oncology clinical trials do not apply in the current scientific and clinical environment of pediatric oncology and drug development; we provide specific recommendations for how the inclusion of children in early-phase investigational cancer drug trials might be accomplished. Automatic inclusion of pediatric patients is appropriate in early-phase trials that assess dose, safety, and pharmacokinetics in a variety of tumor types and later phase trials that assess efficacy in a specific disease that spans adult and pediatric populations.

\section{Conclusion}

Including children in appropriately designed adult clinical oncology trials is feasible and can be done in a way that enhances their access to these agents without compromising safety or development strategies.

\section{J Clin Oncol 35:3781-3787. (C) 2017 by American Society of Clinical Oncology}

\section{INTRODUCTION}

Although major progress has been made in the treatment and even cure of some pediatric cancers, other pediatric cancers, particularly if metastatic at diagnosis, are associated with unacceptably low survival rates based on inadequate existing treatment options and available drugs. ${ }^{1}$ Cancer remains the leading cause of death from disease in children, ${ }^{2}$ with approximately 2,000 children dying from cancer each year in the United States. ${ }^{3}$ Many children who do survive experience a spectrum of short- and long-term toxicities, including cognitive deficits, growth and endocrine dysfunction, infertility, and a risk of developing secondary cancers. ${ }^{4,5}$ There is substantial unmet need for more effective and less toxic agents in children with cancer.

Cancer drug development has been transformed in recent years by rapid advances in biomedical science and technology, and drug development in children has leveraged advances made in adult cancer. To date, children have benefitted less from these advances, because few new drugs are specifically developed for pediatric cancers and initiation of pediatric phase I trials is generally undertaken after extensive testing in adults, well after completion of one or more adult clinical trials, or sometimes not at all. ${ }^{6}$ Meanwhile, many adult oncology clinical trials exclude 
pediatric patients by specifying 18 years as the minimum age of eligibility. Access to some agents for pediatric patients may come first in the form of off-label treatment only after these drugs have been approved for use in adults. Off-label use creates a situation where children may be receiving a drug for which there is no pediatric-specific information about dose, safety, and efficacy or for which long-term effects are not known. This situation further impedes the acquisition of such information because data are not systematically collected or evaluated as a part of off-label treatment. Accrual of patients to pediatric trials and successful completion of trials evaluating drugs whose superior efficacy has already been established in adults can be challenging once a drug is available on the market. This issue is particularly challenging in cancers such as melanoma, some sarcomas, and lymphomas because they occur in both pediatric and adult patients.

As the molecular mechanisms of action of new agents have become more precisely defined, the oncology community is increasingly prioritizing application of scientifically based, clinically relevant approaches to selection of eligibility criteria. ${ }^{7}$ Taking this approach will result in criteria that are not unnecessarily restrictive and can help improve trial accrual and access and the applicability of trial results to real-world patients, which has been recognized as a priority. ${ }^{8}$

\section{PROCESS}

A multistakeholder workshop was convened in May 2016 by ASCO and Friends of Cancer Research to identify opportunities where it is scientifically appropriate to expand trial eligibility. Four working groups composed of patient advocates, drug and biotechnology manufacturers, investigators, and regulators were convened to address the following topics: brain metastases, HIV/AIDS, organ dysfunction, and minimum age for enrollment. Each working group participated in a series of teleconferences in advance of the meeting with the charge to develop specific recommendations based on the state of the science and regulatory guidelines in pediatric oncology and in drug development. This working group was convened to determine when and how the minimum age of eligibility may safely be lowered to younger than age 18 years for adult oncology clinical trials. Herein, we examine the barriers, both real and perceived, that traditionally have prevented patients younger than age 18 years from enrolling in adult oncology clinical trials and discuss how some of these barriers can be overcome. We conclude that many of the historical concerns about including children early in oncology clinical trials do not apply in the current scientific and clinical environment of pediatric oncology and drug development; we provide specific recommendations for how the inclusion of children in early-phase investigational cancer drug trials might be accomplished.

This working group acknowledges that there may be unique safety and/or efficacy signals in children and that children may have different toxicity or drug tolerance and administration profiles compared with adult patients, as has been seen with the use of fenretinide. ${ }^{9,10}$ Nevertheless, we conclude that it is preferable to evaluate new agents in the preapproval setting rather than relying on postmarketing surveillance or off-label use of a new cancer therapy in children.
GENERAL RECOMMENDATIONS FOR INCLUSION OF PEDIATRIC AND ADOLESCENT PATIENTS

The Minimum Age Working Group recommends the following to mitigate risks and facilitate inclusion of pediatric and adolescent patients in general:

1. Adult protocols on which children may be enrolled should include pediatric oncologists as investigators to provide expertise and help address logistical issues. These issues may arise because clinical care of and research involving children occurs primarily at academic pediatric institutions, which most often do not admit adult patients or conduct adult clinical trials.

2. Trials involving children should use a central institutional review board and/or inclusion of pediatric expertise on the institutional review board or ethics committees of record to help educate and support the committee members and assist in review of such studies.

3. The inclusion of established pediatric centers with drug development expertise and infrastructure would help mitigate the operational and regulatory burden and lack of experience that might otherwise exist within a primarily adult clinical center.

4. Young children and any patient with oral or esophageal kinetic dysfunction may not be able to swallow tablets or capsules. Development of either bioavailable extemporaneous compounding of existing agents or pediatric- or adult-friendly oral drug formulations for these populations should be considered early; otherwise, unnecessary delay in pediatric evaluation will occur. If there is sufficient reason to believe a new agent will have potential application to a pediatric population or to adult patients who have similar needs for liquid formulations, the oral or liquid formulation should be tested earlier. Testing of liquid formulations to determine bioavailability when delivered through nasogastric or gastrostomy tubes would be a second consideration for these compounds, as tube composition may affect pharmacokinetics or dosing recommendations.

\section{SPECIFIC SCENARIOS FOR INCLUSION OF PEDIATRIC AND ADOLESCENT PATIENTS AND RECOMMENDED TEMPLATE LANGUAGE}

There are two specific trial scenarios in which the automatic exclusion of pediatric patients are appropriately challenged. These are early-phase trials that assess dose, safety, and pharmacokinetics in a variety of tumor types and later phase trials that assess efficacy in a specific disease that spans adult and pediatric populations, such as chronic myelogenous leukemia, Philadelphia chromosomepositive acute lymphoblastic leukemia, melanoma, Hodgkin lymphoma, and some sarcomas (Table 1). Recommendations for these scenarios are described in the following sections.

\section{Early-Phase Adult Trials That Assess Dose, Safety, and Pharmacokinetics in a Variety of Tumor Types Should Include Children in Certain Circumstances}

Summary. Shared oncogenic pathways or molecular alterations responsible for the etiology of different adult and some pediatric cancers may, depending on the mechanism of drug action, provide a rationale for testing that drug in pediatric patients as early as the phase I stage of testing in adults. Evidence of activity of an investigational drug in one or more pediatric tumor preclinical models could justify early pediatric evaluation, as may activity in adult patients with that same diagnosis or with a disease that shares the same molecular or biologic driver. The driving oncogenic mutation may be appropriately targeted by the same agent, although 
Table 1. Possible Diseases That Could Be Studied (nearly) Simultaneously in Children and Adults

\begin{tabular}{|c|c|c|}
\hline Disease & $\begin{array}{l}\text { Molecular Target, } \\
\text { Driver, or Mutation }\end{array}$ & Comparisons Between Children and Adults \\
\hline CML and Ph-positive ALL & $B C R-A B L$ & $\begin{array}{l}\text { Disease biology is similar, although not identical, between children } \\
\text { and adults with } C M L \text { and Ph-positive ALL. Targeting } B C R-A B L \text { has } \\
\text { been shown to induce remissions in both diseases regardless of } \\
\text { patient age. }\end{array}$ \\
\hline Ph-like leukemias & Various & $\begin{array}{l}\text { Multiple abnormalities have been identified in Ph-like leukemias, } \\
\text { many of which are or may be responsive to small-molecule } \\
\text { inhibitors currently available, as well as newer targets in } \\
\text { development. Examples include JAK, MEK, IL7 receptor, } \\
\text { and CSF1. }\end{array}$ \\
\hline Acute promyelocytic leukemia & $P M L-R A R-\alpha$ & $\begin{array}{l}\text { Differentiation therapies seem to have similar efficacy and toxicity in } \\
\text { children and adults. }\end{array}$ \\
\hline FLT3-positive acute myelogenous leukemia & FLT3 mutation & $\begin{array}{l}\text { FLT3-targeted agents are effective in inducing remission in children } \\
\text { and adults with FLT3-positive acute myelogenous leukemia with } \\
\text { similar toxicity profiles. }\end{array}$ \\
\hline Ewing sarcoma & EWS-FL/1 & Similar pathology and driving mutation seen across the age spectrum. \\
\hline Hodgkin Lymphoma & CD30 & $\begin{array}{l}\text { Similar pathology and activity with anti-CD30 therapies regardless of } \\
\text { patient age. }\end{array}$ \\
\hline Anaplastic large-cell lymphoma & CD30 & $\begin{array}{l}\text { Similar pathology and activity with anti-CD30 therapies regardless of } \\
\text { patient age. }\end{array}$ \\
\hline Melanoma & $B R A F, C T L A-4$ & $\begin{array}{l}\text { Early evidence is that children with melanoma have a lower incidence } \\
\text { of BRAF mutations, although they can respond to BRAF inhibitors. } \\
\text { Similarly, children with melanoma treated with CTLA-4-targeted } \\
\text { agents have similar responses and toxicities to adults treated with } \\
\text { the same agents. }\end{array}$ \\
\hline Neuroblastoma, sarcomas & NTRK fusions & $\begin{array}{l}\text { Substantial response rates have been noted in early-phase clinical } \\
\text { trials targeting NTRK fusion regardless of age of patient and } \\
\text { histopathologic diagnosis reported. }\end{array}$ \\
\hline
\end{tabular}

Abbreviations: ALL, acute lymphoblastic leukemia; CML, chronic myelogenous leukemia; Ph, Philadelphia chromosome.

in different tumor types in adults compared with children. Examples of this include an ALK inhibitor that may be used in adults for non-small-cell lung cancer but should be tested in children with ALK-positive anaplastic large-cell lymphoma or neuroblastoma. ${ }^{11-13}$

Recommendation. We recommend that study of a drug in a specific pediatric population could be conducted when there is scientific rationale to suggest that children with a specific diagnosis may benefit and when there is adequate nonclinical or clinical information to sufficiently mitigate patient risk. When such rationale exists, prospective inclusion of a pediatric-specific dose-escalation cohort within a larger adult trial should be considered, with the objectives of defining pediatric dose-limiting toxicities and recommended dose, as well as assessing safety and pharmacokinetics in younger patients. Generally, opening enrollment of a pediatric cohort in the phase I setting should occur when sufficient data in adults exist to guide dosing and toxicity monitoring, perhaps just before any cohort expansion at the recommended phase II dose. Pediatric patients may experience different dose-limiting toxicities and adverse event profiles than adults. Alternatively, a pediatric cohort could be treated as a separate stratum and escalated independently of adults until a dose appropriate for the specific age group is defined.

Younger age groups present additional considerations; therefore, it may be appropriate to use staged enrollment starting with older children once initial adult safety and toxicity data are known. For example, patients age 12 to 17 years could be enrolled first, because they are most likely to be physiologically like adult patients and are expected to tolerate dosing in a similar fashion, and then those age 6 to 11 years could be enrolled, followed by even younger children, as appropriate to the epidemiology of the disease(s) under study. Organ function, maturation of metabolic pathways, and body-surface area all change rapidly over time in young children; however, in many cases, children can receive the same weightbased or body-surface area-based doses as adults. ${ }^{14}$

Younger children may be at risk for developmental toxicities with certain drugs that would not have been identified in adults, but often, the classes of drugs with potential developmental toxicities are identifiable given the specific molecular targets or signaling pathways affected by the drug, ${ }^{15}$ and protocols should include a longer period of follow-up to better assess toxicities when possible. Although this is not always easy, it is critical to be able to assess multiple parameters that may differ when newer agents are introduced to children and that may not be evident in adult patients.

\section{Sample template for inclusion criteria.}

1. Adolescent/pediatric patients age [protocol author to insert age minimum and maximum specific to the study under consideration] will be included after enrollment of adult patients once safety and toxicity have been established. Participating sites will be notified when adolescent/pediatric patient enrollment may begin.

2. Adolescent/pediatric patients age [protocol author to insert age minimum and maximum specific to the study under consideration] will be included starting one dose cohort behind the current adult cohort in which there are no dose-limiting toxicities identified. Participating sites will be notified when enrollment to the adolescent/pediatric stratum may begin.

3. Adolescent/pediatric patients age [protocol author to insert age minimum and maximum specific to the study under consideration] will be included in age-specific cohorts that will be staggered starting one dose cohort behind the current adult cohort in which there are no dose-limiting toxicities identified. 
Participating sites will be notified when each adolescent/ pediatric cohort enrollment may begin.

4. Adolescent/pediatric patients age [protocol author to insert age minimum and maximum specific to the study under consideration] are included in this trial in a separate cohort that will accrue simultaneously with the adult cohort [specify age 18 and older or protocol-specific upper age limit].

\section{Later Stage Trials That Assess Efficacy in a Specific Disease That Spans Adult and Pediatric Populations, Such as Chronic Myelogenous Leukemia, Philadelphia Chromosome-Positive Acute Lymphoblastic Leukemia, Melanoma, Hodgkin Lymphoma, and Some Sarcomas, Could Enroll Simultaneous/y With Adult and Pediatric Cohorts}

Summary. Currently, the front-line Children's Oncology Group trials for acute lymphoblastic leukemia include patients up to age 30 years, and some Ewing sarcoma and osteosarcoma trials allow patients up to age 40 or 50 years. These age considerations are made based on the biology of the disease and the age distribution of the patients affected by the diseases. For example, EWS-FLI1 and related fusions are present in the vast majority of, if not all, Ewing sarcomas. ${ }^{16-18}$ As such, an agent targeting EWS-FLI1 should be tested in patients with that fusion regardless of age if the hypothesis is that disease activity is based on the fusion rather than the age of the patient in which it is tested. Similarly, for the anti-CD30-targeted agents, patients with Hodgkin lymphoma or anaplastic large-cell lymphoma can be tested regardless of age, because the disease spans age ranges but the driving tumor biology is similar. ${ }^{19-23}$ Additional examples include the CD19-directed bispecific T-cell engager blinatumomab and the myriad chimeric antigen receptor T-cell therapy trials that have shown both efficacy and similar safety profiles in children and adults with CD19-positive disease, both acute lymphoblastic leukemia and non-Hodgkin lymphoma. ${ }^{24-32}$

Recommendation. We recommend that the age range of patients enrolled onto later phase, disease-specific trials should reflect the age range of patients with that disease. We recommend that latestage trials for diseases that span the pediatric and adult patient populations routinely include patients 12 years of age and older on the basis of the similarity in drug metabolism and excretion between adults and postpubertal adolescents. Where growth and development could be adversely affected based on nonclinical or early clinical data, a more restrictive age cutoff may be appropriate or more stringent monitoring may be incorporated. In some cases, it may also be appropriate to include patients younger than age 12 years. In essence, the minimum age of eligibility specified in late-phase trials should be tailored to the biology of the disease under study, the scientific objectives of the trial, and the existing data regarding the mechanism of action and safety profile of the drug.

Template for inclusion criteria. Adolescent patients age 12 years and older are allowed with signed assent and parental consent according to institutional guidelines and requirements.

\section{DISCUSSION}

Children have historically been excluded from first-in-human studies of promising new cancer drugs and later phase adult clinical trials, even for cancers that occur in both adults and children. Development of new cancer drugs for children leverages discovery and development of drugs for adults with cancer. Thus, clinical trials of novel therapies for children are either delayed or never undertaken, and pediatric patients with cancer can only access these new drugs off-label for which no dosing information is provided. Offlabel use eliminates the opportunity to collect data on safe and effective use of drug products in other children who might potentially benefit or be spared from the toxicity of an ineffective drug.

Despite progress in recent years, children with cancer need more timely discovery, access to, and evaluation of new investigational drugs. To spur pediatric drug development, two legislative programs have been implemented in the United States. ${ }^{33}$ The Pediatric Research Equity Act (PREA) requires new drug applications and biologics license applications or supplements to applications for an adult indication to contain information from one or more studies for the same indication in pediatric patients, unless the applicant has obtained a waiver or deferral. ${ }^{34,35}$ However, PREA does not apply to indications for which a drug has received orphan designation (for indications affecting $<200,000$ people per year in the United States). Many cancer types, including those that span adult and pediatric populations, fall below this threshold. Moreover, the most common adult cancers, such as lung, breast, colorectal, and prostate cancer, would not be eligible for orphan designation because they do not occur with any frequency in children and pediatric evaluations; therefore, PREA requirements are waived. For these reasons, there has never been a PREA requirement for pediatric evaluation of a cancer drug. A significant recent advance is that Congress is considering legislation (the Research to Accelerate Cures and Equity for Children Act, H.R. 231/S. 456) that would modify PREA to address these problems. The legislation will apply the PREA requirements to drugs that receive an orphan drug designation and require pediatric testing if the molecular target of the drug is substantively relevant to pediatric cancers.

The United States also provides an incentive for pediatric drug development through the Best Pharmaceuticals for Children Act (BPCA), which allows for 6 months added market exclusivity if specific pediatric research agreed upon by the US Food and Drug Administration (FDA) and drug sponsor is completed. ${ }^{36}$ However, even with the incentives of the PREA and BPCA, new strategies to promote and facilitate earlier investigation of oncology drugs in children are needed. Here, we recommend the inclusion of pediatric patients with cancer in adult clinical trials when appropriate. Even with this approach, pediatric-focused clinical trials will continue to be necessary, particularly for cancer types that occur exclusively in pediatric patients.

Although pediatric oncology drug development is complicated by many factors (Table 2), such as the rarity of pediatric cancers and additional ethical considerations and regulations for vulnerable populations, the prevailing pattern of excluding children from adult trials is derived largely from a concern for safety even when there may be reason to believe that an individual child could benefit from an agent being studied in an adult population. Historically, the tendency has been to protect children from research that may carry unknown risks rather than provide the potential for benefit to children through research, which, in some cases, has led to overprotection at the expense of access to a promising agent. 
Table 2. Challenges in Pediatric Drug Development

\begin{tabular}{|c|c|}
\hline Challenge & Considerations and Suggestions for Improvement \\
\hline $\begin{array}{l}\text { Too many new agents to study } \\
\text { within reasonable time frames }\end{array}$ & $\begin{array}{l}\text { - Could the scientific objectives be achieved in any other way that is either more efficient or less restrictive? Novel study } \\
\text { design and limited numbers of dose cohorts and patients per cohort can reduce numbers of patients enrolled per study. } \\
\text { - Are multiple trials needed if one could suffice? } \\
\text { - Consider agents with similar mechanisms of action and ensure that duplicative studies are not being conducted without } \\
\text { benefit or advancement of scientific understanding. } \\
\text { - Are separate pediatric trials needed? }\end{array}$ \\
\hline Safety and toxicity & $\begin{array}{l}\text { - Is patient safety being adequately protected? } \\
\text { - Are potential toxicities and mechanisms of action accounted for and followed for the appropriate length of time? } \\
\text { - Could postmarketing reporting be extended or altered to accommodate unique mechanisms of action or toxicities? } \\
\text { - Does limiting or restricting protocol inclusion and exclusion criteria support or hinder the scientific goal(s) of the study? }\end{array}$ \\
\hline
\end{tabular}

Although the appearance of risk associated with a new drug may be amplified if a child experiences a serious adverse reaction to an investigational drug, we believe this is a perceived risk, rather than a real risk. There is concern among some that a high-profile adverse event in a pediatric patient could jeopardize the development of a new drug, ultimately limiting access to an effective therapy for a broader patient population. However, adverse events in pediatric patients have not impeded development of any oncology drug reviewed at the FDA, given all available evidence to date (G. Reaman, personal communication, May 2016). In fact, the FDA encourages the early design and conduct of pediatric trials with investigational agents or the inclusion of pediatric patients in certain adult clinical trials when appropriate to expedite the development of safe and effective therapies to treat cancer in children. ${ }^{37}$

In the approach we propose here, a serious adverse event in the pediatric population may appropriately interrupt or halt development in that population without impacting drug development in adults unless there is evidence that the safety signal may also apply to adults. Conversely, should pediatric patients tolerate a higher dose than adults, our recommendations will facilitate identification of that scenario and the most appropriate dosing in each patient population.

Not addressed here, but critical to the success of trials spanning a wider age range, are novel clinical trial designs that are more efficient and involve the fewest patients needed to achieve trial goals while simultaneously providing the best patient safety parameters. Indeed, the trial modifications proposed herein could require different analyses by cohort based on such designs.

Discussing reasons for why a pharmaceutical sponsor may choose to include or exclude children in early-phase trials using the recommendations we propose is beyond the scope of this article. The development prioritization for sponsors is typically focused on the most rapid path to approval of an agent that can reach the largest patient population once commercialized. Clearly, the market for pediatric cancers overall is a small one compared with adult oncology indications, and it would be the rare disease or indication that would prove to be commercially successful in children. Dinutuximab for neuroblastoma is a successful example of the use of pediatric disease priority review vouchers as a strategy to increase enrollment onto clinical trials by including the pediatric and adolescent patient populations. ${ }^{38}$ Approval of pembrolizumab for solid tumors with a high-level microsatellite instability or mismatch repair deficient biomarker provides an example of trials that enrolled both adult and pediatric patients with a common biomarker. ${ }^{39}$ Inclusion of pediatric patients in the trial provided simultaneous approval in adult and pediatric patients, in addition to being the first approval for all solid tumor types.

Children with cancer clearly stand to benefit from earlier investigation of novel agents. Drug sponsors stand to benefit as well. If sufficient numbers of pediatric patients are enrolled, they may provide meaningful information that can lead to early identification of drugs with a strong signal of antitumor activity against one or more cancers in children that should be studied further. An example of the success of early inclusion of pediatric patients was recently presented by Federman et $\mathrm{al}^{40}$ and Hyman et $\mathrm{al}^{41}$ and at the 2017 ASCO Annual Meeting. On the basis of early data demonstrating prolonged survival and a favorable adverse effect profile for the drug larotrectinib (a tropomyosin kinase receptor inhibitor) in adults with NTRK fusions, a phase I/II study was initiated in children harboring NTRK fusions. ${ }^{40}$ The adult and pediatric trials were conducted simultaneously. Combined analysis of the trials reported an overall response rate of $78 \%$ in 12 unique tumor sites, with efficacy observed in both populations, as well as tolerability. ${ }^{41}$ The safety and pharmacokinetic information derived from the study of cancer therapies in pediatric patients enrolled onto adult clinical trials can be used to help fulfill the terms of a Pediatric Written Request and can provide useful information for product labeling. Full adoption of these recommendations will require the engagement of all stakeholders, 
including patients and families, investigators, the pharmaceutical industry, regulators, advocacy groups, and the institutional review boards tasked with protecting patient safety. This will be an organic process that requires regular review and revision within the context of the rapidly evolving drug development environment.

Finally, because clinical trials are increasingly being conducted globally, engagement of and coordination with international regulatory authorities will be necessary to assist sponsors in developing strategies that meet regulatory requirements within and outside the United States. Deeper and more frequent international collaborations, harmonization of regulatory processes where appropriate, and support and continued cooperation and advocacy from all stakeholders will be required.

\section{CONCLUSION}

Automatic inclusion of pediatric patients is appropriate in earlyphase trials that assess dose, safety, and pharmacokinetics in a variety of tumor types and in later phase trials that assess efficacy in a specific disease that spans adult and pediatric populations. Sponsors, treating institutions, and funding agencies will be tasked with the duty of addressing the logistical processes and procedural hurdles to accommodate the inclusion of younger patients in clinically and scientifically appropriate clinical trials without jeopardizing the trial conduct. We must continue to work collaboratively to enhance the value of each trial conducted, because rapid technologic advances continue to outpace our current trial structure and capacities, and ultimately to improve the landscape for the patients who need new treatments the most. With continued communication, understanding, and collaboration among all stakeholders and the ability to study diseases and outcomes of treatment more carefully, pediatric patients with cancer can fully benefit from the great strides currently being made to conquer cancer.

\section{AUTHORS' DISCLOSURES OF POTENTIAL CONFLICTS OF INTEREST}

Disclosures provided by the authors are available with this article at jco.org.

\section{AUTHOR CONTRIBUTIONS}

Conception and design: Lia Gore, S. Percy Ivy, Frank M. Balis, Eric Rubin, Katherine Thornton, Martha Donoghue, Samantha Roberts, Suanna Bruinooge, Nancy Goodman, Caroline Schenkel, Gregory Reaman

Administrative support: Samantha Roberts, Suanna Bruinooge, Caroline Schenkel

Collection and assembly of data: Lia Gore, S. Percy Ivy, Frank M. Balis, Eric Rubin, Katherine Thornton, Martha Donoghue, Samantha Roberts, Suanna Bruinooge, Nancy Goodman, Gregory Reaman

Data analysis and interpretation: Lia Gore, S. Percy Ivy, Frank M. Balis, Eric Rubin, Katherine Thornton, Martha Donoghue, Samantha Roberts, Suanna Bruinooge, Jennifer Ersek, Nancy Goodman, Gregory Reaman

Manuscript writing: All authors

Final approval of manuscript: All authors

Accountable for all aspects of the work: All authors

\section{REFERENCES}

1. Adamson PC: Improving the outcome for children with cancer: Development of targeted new agents. CA Cancer J Clin 65:212-220, 2015

2. Heron M: Leading causes for 2010. Natl Vital Stat Rep 62:1-96, 2013

3. Smith M, Ries L, Gurney J: Cancer incidence and survival among children and adolescents: United States SEER Program 1975-1995. https://seer.cancer. gov/archive/publications/childhood/childhood-monograph. pdf

4. Hudson MM, Mertens $A C$, Yasui $Y$, et al: Health status of adult long-term survivors of childhood cancer: A report from the Childhood Cancer Survivor Study. JAMA 290:1583-1592, 2003

5. Bottomley SJ, Kassner E: Late effects of childhood cancer therapy. J Pediatr Nurs 18:126-133, 2003

6. Alliance for Childhood Cancer, American Cancer Society: Translating Discovery into Cures for Children with Cancer: Childhood Cancer Research Landscape Report. http://www.cancer.org/acs/groups/content/ @editorial/documents/document/acspc-048347.pdf

7. Kim ES, Bernstein D, Hilsenbeck SG, et al: Modernizing eligibility criteria for molecularly driven trials. J Clin Oncol 33:2815-2820, 2015

8. Beaver JA, Ison G, Pazdur R: Reevaluating eligibility criteria: Balancing patient protection and participation in oncology trials. N Engl J Med 376: 1504-1505, 2017

9. Garaventa A, Luksch R, Lo Piccolo MS, et al: Phase I trial and pharmacokinetics of fenretinide in children with neuroblastoma. Clin Cancer Res 9 2032-2039, 2003

10. Puduvalli VK, Yung WKA, Hess $K R$, et al: Phase II study of fenretinide (NSC 374551) in adults with recurrent malignant gliomas: A North American Brain Tumor Consortium study. J Clin Oncol 22 4282-4289, 2004

11. Camidge DR, Bang YJ, Kwak EL, et al: Activity and safety of crizotinib in patients with ALKpositive non-small-cell lung cancer: Updated results from a phase 1 study. Lancet Oncol 13: 1011-1019, 2012

12. Balis FM, Thompson PA, Mosse YP, et al: First-dose and steady-state pharmacokinetics of orally administered crizotinib in children with solid tumors: A report on ADVL0912 from the Children's Oncology Group phase 1/pilot consortium. Cancer Chemother Pharmacol 79:181-187, 2017

13. Mossé YP, Lim MS, Voss SD, et al: Safety and activity of crizotinib for paediatric patients with refractory solid tumours or anaplastic large-cell lymphoma: A Children's Oncology Group phase 1 consortium study. Lancet Oncol 14:472-480, 2013

14. Lee DP, Skolnik JM, Adamson PC: Pediatric phase I trials in oncology: An analysis of study conduct efficiency. J Clin Oncol 23:8431-8441, 2005

15. Gore L, DeGregori J, Porter CC: Targeting developmental pathways in children with cancer: What price success? Lancet Oncol 14:e70-e78, 2013

16. May WA, Gishizky ML, Lessnick SL, et al: Ewing sarcoma 11;22 translocation produces a chimeric transcription factor that requires the DNAbinding domain encoded by FLI1 for transformation. Proc Natl Acad Sci USA 90:5752-5756, 1993
17. Dagher R, Pham TA, Sorbara $L$, et al: Molecular confirmation of Ewing sarcoma. J Pediatr Hematol Oncol 23:221-224, 2001

18. de Alava E, Kawai A, Healey JH, et al: EWSFLI1 fusion transcript structure is an independent determinant of prognosis in Ewing's sarcoma. J Clin Oncol 16:1248-1255, 1998

19. Cheng J, Zhu H, Choi JK: CD30 expression in pediatric neoplasms, study of 585 cases. Pediatr Dev Pathol 20:191-196, 2017

20. Pierce JM, Mehta A: Diagnostic, prognostic and therapeutic role of CD30 in lymphoma. Expert Rev Hematol 10:29-37, 2017

21. Alperovich $A$, Younes $A$ : Targeting CD30 using brentuximab vedotin in the treatment of Hodgkin Iymphoma. Cancer J 22:23-26, 2016

22. $\mathrm{Xu} \mathrm{ML}$, Acevedo-Gadea C, Seropian S, et al: Expression of CD30 as a biomarker to predict response to brentuximab vedotin. Histopathology 69: 155-158, 2016

23. Hapgood G, Savage KJ: The biology and management of systemic anaplastic large cell lymphoma. Blood 126:17-25, 2015

24. Martinelli G, Boissel N, Chevallier $P$, et al: Complete hematologic and molecular response in adult patients with relapsed/refractory Philadelphia chromosome-positive B-precursor acute lymphoblastic leukemia following treatment with blinatumomab: Results from a phase II, single-arm, multicenter study. $\mathrm{J}$ Clin Oncol 35:1795-1802, 2017

25. Gökbuget $N$, Zugmaier G, Klinger $M$, et al: Long-term relapse-free survival in a phase 2 study of blinatumomab for the treatment of patients with minimal residual disease in B-lineage acute 
lymphoblastic leukemia. Haematologica 102:e132-e135 2017

26. Topp MS, Gökbuget N, Stein AS, et al: Safety and activity of blinatumomab for adult patients with relapsed or refractory B-precursor acute lymphoblastic leukaemia: A multicentre, single-arm, phase 2 study. Lancet Oncol 16:57-66, 2015

27. von Stackelberg A, Locatelli F, Zugmaier G, et al: Phase I/phase II study of blinatumomab in pediatric patients with relapsed/refractory acute lymphoblastic leukemia. J Clin Oncol 34:4381-4389, 2016

28. Maude SL, Frey N, Shaw PA, et al: Chimeric antigen receptor $\mathrm{T}$ cells for sustained remissions in leukemia. N Engl J Med 371:1507-1517, 2014

29. Lee DW, Kochenderfer JN, Stetler-Stevenson M, et al: T cells expressing CD19 chimeric antigen receptors for acute lymphoblastic leukaemia in children and young adults: A phase 1 dose-escalation trial. Lancet 385:517-528, 2015

30. Gardner RA, Finney $O$, Annesley $C$, et al: Intent-to-treat leukemia remission by CD19 CAR $T$ cells of defined formulation and dose in children and young adults. Blood 129:3322-3331, 2017
31. Gill S, Porter DL: CAR-modified anti-CD19 $T$ cells for the treatment of B-cell malignancies: Rules of the road. Expert Opin Biol Ther 14:37-49, 2014

32. Locke FL, Neelapu SS, Bartlett NL, et al: Phase 1 results of ZUMA-1: A multicenter study of KTE-C19 anti-CD19 CAR T cell therapy in refractory aggressive lymphoma. Mol Ther 25: 285-295, 2017

33. Institute of Medicine: Safe and Effective Medicines for Children: Pediatric Studies Conducted Under BPCA and PREA. Washington, DC, The National Academies Press, 2012

34. Field MJ, Boat TF: Safe and Effective Medicines for Children: Pediatric Studies Conducted Under the Best Pharmaceuticals for Children Act and the Pediatric Research Equity Act. Washington, DC, The National Academies Press, 2012

35. US Food and Drug Administration: Draft guidance for industry: How to comply with the Pediatric Research Equity Act. http://www.fda.gov/ downloads/drugs/guidancecomplianceregulatory information/guidances/ucm079756.pdf
36. Best Pharmaceuticals for Children Act (January 4, 2002) Pub Law 107-109, 107th Congress

37. Chuk MK, Mulugeta $Y$, Roth-Cline $M$, et al: Enrolling adolescents in disease/target-appropriate adult oncology clinical trials of investigational agents. Clin Cancer Res 23:9-12, 2017

38. US Food and Drug Administration: FDA approves first therapy for high-risk neuroblastoma. https://www.fda.gov/newsevents/newsroom/press announcements/ucm437460.htm

39. US Food and Drug Administration: FDA approves first cancer treatment for any solid tumor with a specific genetic feature. https://www.fda.gov/NewsEvents/ Newsroom/PressAnnouncements/ucm560167.htm

40. Federman N, Albert CM, Turpin B, et al: Phase $1 / 2$ study of the selective TRK inhibitor larotrectinib in pediatric patients with cancer. J Clin Oncol 35, 2017 (suppl; abstr TPS10577)

41. Hyman DM, Laetsch TW, Kummar S, et al: The efficacy of larotrectinib (LOXO-101), a selective tropomyosin receptor kinase (TRK) inhibitor, in adult and pediatric TRK fusion cancers. J Clin Oncol 35, 2017 (suppl; abstr LBA2501)

\section{Affiliations}

Lia Gore, Children's Hospital Colorado and University of Colorado School of Medicine, Aurora, CO; S. Percy Ivy, National Cancer Institute, Bethesda; Martha Donoghue and Gregory Reaman, US Food and Drug Administration, Silver Spring, MD; Frank M. Balis, Children's Hospital of Philadelphia, Philadelphia, PA; Eric Rubin, Merck Research Laboratories, Kenilworth, NJ; Katherine Thornton, Dana-Farber Cancer Institute, Boston, MA; Samantha Roberts, Friends of Cancer Research and Genentech; Nancy Goodman, Kids v Cancer, Washington, DC; Suanna Bruinooge and Caroline Schenkel, ASCO, Alexandria, VA; and Jennifer Ersek, Levine Cancer Institute, Charlotte, NC. 
Modernizing Clinical Trial Eligibility: Recommendations of the American Society of Clinical Oncology-Friends of Cancer Research Minimum Age Working Group

The following represents disclosure information provided by authors of this manuscript. All relationships are considered compensated. Relationships are self-held unless noted. I = Immediate Family Member, Inst = My Institution. Relationships may not relate to the subject matter of this manuscript. For more information about ASCO's conflict of interest policy, please refer to www.asco.org/rwc or ascopubs.org/jco/site/ifc.

Lia Gore

Employment: ARIAD (I)

Leadership: ARIAD (I)

Stock or Other Ownership: ARIAD (I), Amgen, Sanofi, Celgene, ARIAD, Clovis Oncology, Agios (I)

Honoraria: Amgen

Consulting or Advisory Role: Celgene, MedImmune, Novartis, ProEd Communications, Genentech, Amgen, Medscape

Patents, Royalties, Other Intellectual Property: Patent held for diagnostic discovery and treatment response methodology tools in the use of magnetic resonance spectroscopy for leukemia.

Travel, Accommodations, Expenses: Amgen, Genentech

S. Percy Ivy

No relationship to disclose

Frank M. Balis

Research Funding: United Therapeutics (Inst)

Travel, Accommodations, Expenses: Loxo

Eric Rubin

Employment: Merck

Stock or Other Ownership: Merck

\section{Katherine Thornton}

Consulting or Advisory Role: Novartis

Speakers' Bureau: Novartis

Travel, Accommodations, Expenses: Novartis

Martha Donoghue

No relationship to disclose

Samantha Roberts

Employment: Genentech

Suanna Bruinooge

No relationship to disclose

Jennifer Ersek

No relationship to disclose

Nancy Goodman

No relationship to disclose

Caroline Schenkel

No relationship to disclose

Gregory Reaman

No relationship to disclose 


\section{Acknowledgment}

This article was developed as a consensus document of the Minimum Age Working Group as part of a collaboration between ASCO, Friends of Cancer Research, and the US Food and Drug Administration (FDA). The contents of this article were presented on May 12, 2016, as part of a collaborative workshop. We thank all the participants of the workshop, as well as the planning committee (Eric Rubin, Nancy Roach, and Elizabeth Garret-Mayer), FDA staff (Richard Pazdur, Gwynn Ison, Julia Beaver, Tatiana Prowell, and Raji Sridhara), ASCO staff (Edward S. Kim, Richard L. Schilsky, Suanna Bruinooge, and Caroline Schenkel), and Friends of Cancer Research staff (Ellen Sigal, Jeff Allen, Marina Kozak, and, in particular, Samantha Roberts for leading this working group), for their expertise, thoughtful input on the project and article, and logistical support. 